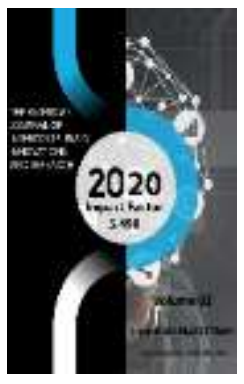

Journal Website: http://usajournalshub.c om/index,php/tajiir

Copyright: Original content from this work may be used under the terms of the creative commons attributes 4.0 licence.

\section{Determination of Sections of General Surfaces of the Second Order on Predetermined Circles}

\author{
Fakhriddin UrolovichToshpulatov \\ Lecturer, Termiz State Universit, Termiz, Uzbekistan \\ Bakhtiyor Karamatovich Mominov \\ Lecturer, Termiz State University, Termiz, Uzbekistan \\ Ilkhomjon Chorshanbeyvich Mamatkulov \\ Lecturer, Termiz State University, Termiz, Uzbekistan
}

\title{
ABSTRACT
}

This article discusses how to define cross-sections of general second-order surfaces on predefined circles. It also describes the work being done to create the necessary conditions for boys and girls to acquire modern knowledge and grow spiritually.

\section{KEYWORDS}

Common surface, radius, plane, axial ellipsoid, elliptical cone, theorems, circle, parallel.

\section{INTRODUCTION}

In our country, special attention is paid to the upbringing of the young generation. Efforts are being made to create the necessary conditions for boys and girls to receive a modern education and grow up spiritually.
In particular, this work and research is carried out in the field of descriptive geometry, drawing, as well as in other areas. 


\section{THE MAIN RESULTS AND CONCLUSION}

It is well known that solving positional and metric problems associated with second-order surfaces and determining the state of predetermined second-order curves can be considered one of the most important issues in determining various parts of machine mechanisms or construction methods encountered in engineering practice.

One of these tasks is to determine the state of circular sections of radius $R$, predetermined by common second-order surfaces. To do this, it is necessary to determine the position of the intersecting planes on the second-order common surfaces so that they intersect the surfaces along a circle of given radius $R$.

On second-order surfaces, this problem is less difficult to solve. This is because if the surface of a second-order circle is intersected by a plane perpendicular to its axis, it is also possible to determine the position of a circle of radius $R$ between these planes.

In the process of making machine parts, it is necessary to make a line of intersection of the surfaces of the parts. The intersection lines of surfaces consist of a set of points common to surfaces. To create an intersection line, similar points are identified and connected in series.

Auxiliary cutting surfaces are used to construct the intersection line of the surfaces. The position of the auxiliary cutting surface should be chosen so that it intersects the cutting surfaces in straight lines or circles. Plane, spherical, cylindrical or conical surfaces are used as auxiliary cutting surfaces.
At the intersection of a prism and a pyramid, the method of determining the point of intersection of a straight line with a plane is used to determine their line of intersection.

The points of intersection of the edges of the pyramid with the sides of the prism are determined by passing $K, L, M$ and $N, R, R$ to the auxiliary planes. It is obvious that their horizontal projections belong to the projecting sides of the prism.

As a result, if we connect the defined points in order, the resulting broken lines $K, L, M$ and $N$, $T, R, Q, R$ will be the line of intersection of the pyramid and the prism. The visibility and invisibility of the intersection lines depend on the invisibility of the sides of the prism and the pyramid.

If the line of intersection lies on the visible side of the pyramid and the sides of the prism, then it is visible, otherwise it is invisible. It should also be noted that if one of the two sides of the intersection is not visible, the line of intersection at these intersections will also be invisible. These points are made by passing an arbitrary auxiliary plane parallel to the Q plane and a plane $\mathrm{P}$ intersecting the two cylinders.

Horizontal traces $\mathrm{P}_{1} \mathrm{H}, \mathrm{P}_{4} \mathrm{H}$ of the auxiliary planes $\mathrm{P}_{1}, \mathrm{P}_{4}$ intersecting one of the cylindrical surfaces are made. This will be $P_{1} H\|P 4 H\| Q H$. The characteristic points of the line of intersection are found in the same way as the characteristic points of the line of intersection of a cone or a cylinder with a cone. These horizontal projections of points $A\left(A^{\prime}, A^{\prime \prime}\right), B$ $\left(B^{\prime}, B^{\prime \prime}\right), C\left(C^{\prime}, C^{\prime \prime}\right)$ are made using plane traces $\mathrm{P}_{2} \mathrm{H}, \mathrm{P}_{3} \mathrm{H}$. Other intermediate points of the line of intersection are made by passing parallel 
auxiliary planes $\mathrm{P}$. All resulting intersections are joined by a smooth curve. The algorithm for creating the line of intersection of a prism and a cylinder is performed in the above sequence.

If the plane intersects the general surfaces of the second order along a predetermined circle of radius $\mathrm{R}$, then it can be determined that the planes symmetric to this plane also intersect this surface along the circle of radius $\mathrm{R}$. Consequently, a family of such intersecting planes forms a family of two parametric planes.

Graphic-analytical solutions of such problems have been investigated and partially cited in the literature [1].

It is known that circular cuts are also present on general surfaces of the second order. In this article, you can graphically determine the position of intersecting planes on a circle of radius $\mathrm{R}$ with a given radius on common surfaces of order 2 .

Based on the following theorems of Gosard Monge in the field of descriptive geometry and its particular cases, we can assert that the above problem can be solved synthetically.

If two surfaces of order 2 intersect on one straight line, then they intersect on another straight line, and this is also a straight line.

If two surfaces of order 2 intersect at two points, then their lines of intersection are also divided into two straight curves of order two.
To determine the position of a circle of radius $\mathrm{R}$ on the general surfaces of the second order, it is first necessary to determine the position of the intersecting planes on the circles.

For example, Figures 1 and 2 show the directions of the planes intersecting the surfaces of a axial ellipsoid and an elliptical cone in a circle. Based on the above theorems, using the experimental sphere surfaces at points $A\left(A^{\prime \prime}\right)$ and $B\left(B^{\prime \prime}\right)$ of three-axis ellipsoid and elliptical cone surfaces, the possibility of determining the directions of circular sections of general surfaces of the 2 nd order is shown.

In Figure 1, the direction of the projection planes of the profile $G w$ and $G 1 w$ is determined by the direction of the circular sections on the three-axis ellipsoid.

To do this, draw a sphere of arbitrary radius Ro, which points to points $A$ " and $B$ " through point $\mathrm{O}_{1}$ " on the axis of the frontal projection of the surface of this axial ellipsoid.

\section{CONCLUSION}

According to the condition of the theorem, this sphere and axial ellipsoidal surfaces intersect in circles $C$ " ' $D$ ' " and $E$ " ' $F$ ' " passing through points $A$ " 'EB' ", GW and G1w forms the direction of the planes circles. We now determine the position of the circular planes of a given radius $\mathrm{R}$. To do this, first determine the position of the point $M$ "'in the middle of the circle $C^{\prime}$ " $D$ " ', which is the direction of the circle. 


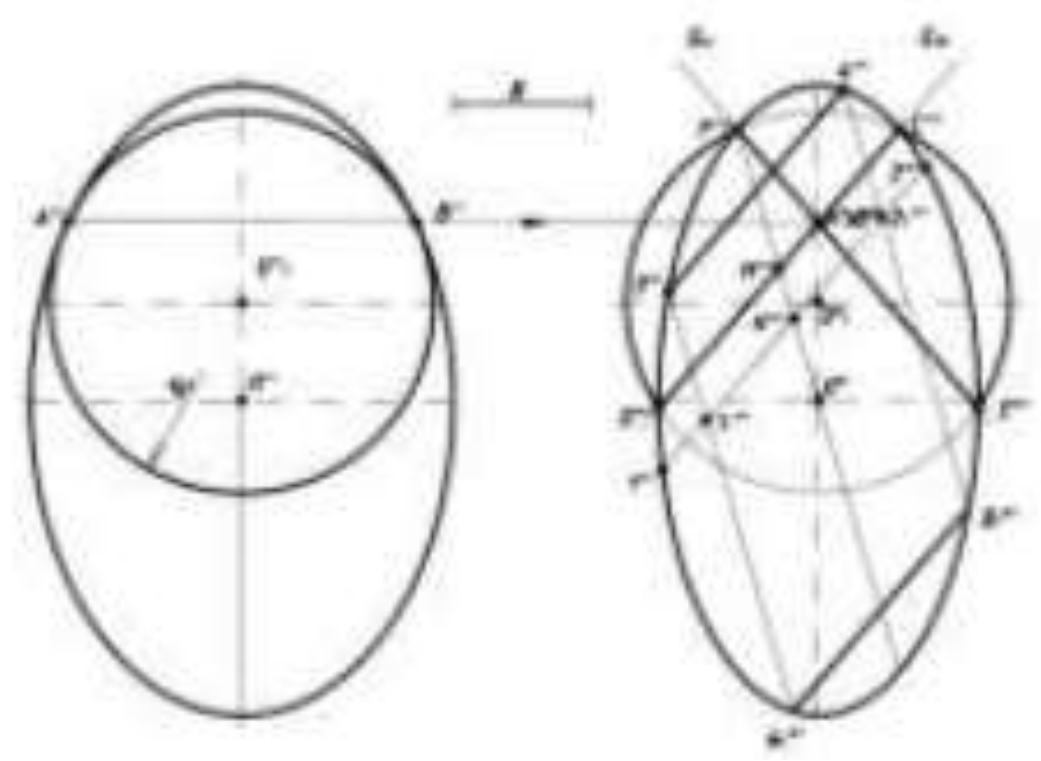

Figure 1.

Then an arbitrary section 1 " ' 2 ' "' is made parallel to the plane of the circle. If the point $N$ " 'in the middle of this section is defined and connected to the point $M^{\prime}$ ", a joint diameter passing through the point $O$ "' 'is formed. The radius $\mathrm{R}$ of a predetermined circle is placed on either side of the point $N$ " ', which belongs to the line $1^{\prime \prime} " 2 "$ '. When lines are drawn parallel to the joint diameter from the points 11 " 'and $21^{\prime \prime}$ ", they intersect the given surface at points 3 "' 'and 4 '".

Connecting these points is $3^{\prime \prime}$ ' 4 ' " = $2 R$ to solve the problem. A circle symmetric to this circle is $41 "$ " 51 ' " = 2R. Similarly, it is possible to determine the position of circles with a predetermined radius $\mathrm{R}$ parallel to the direction Gw.

Based on the above sequences and definitions, the states of the circular planes of radius $R$ defined on the surface of the elliptical cone shown in Figure 2 are determined.

In this case, the "joint diameters" of the straight line connecting the center of the circle pass through the end of the elliptical cone. The states of circles of radius $R$, defined on the surfaces of a second-order elliptic paraboloid, an elliptic hyperboloid, can also be determined by the above graphical methods. 


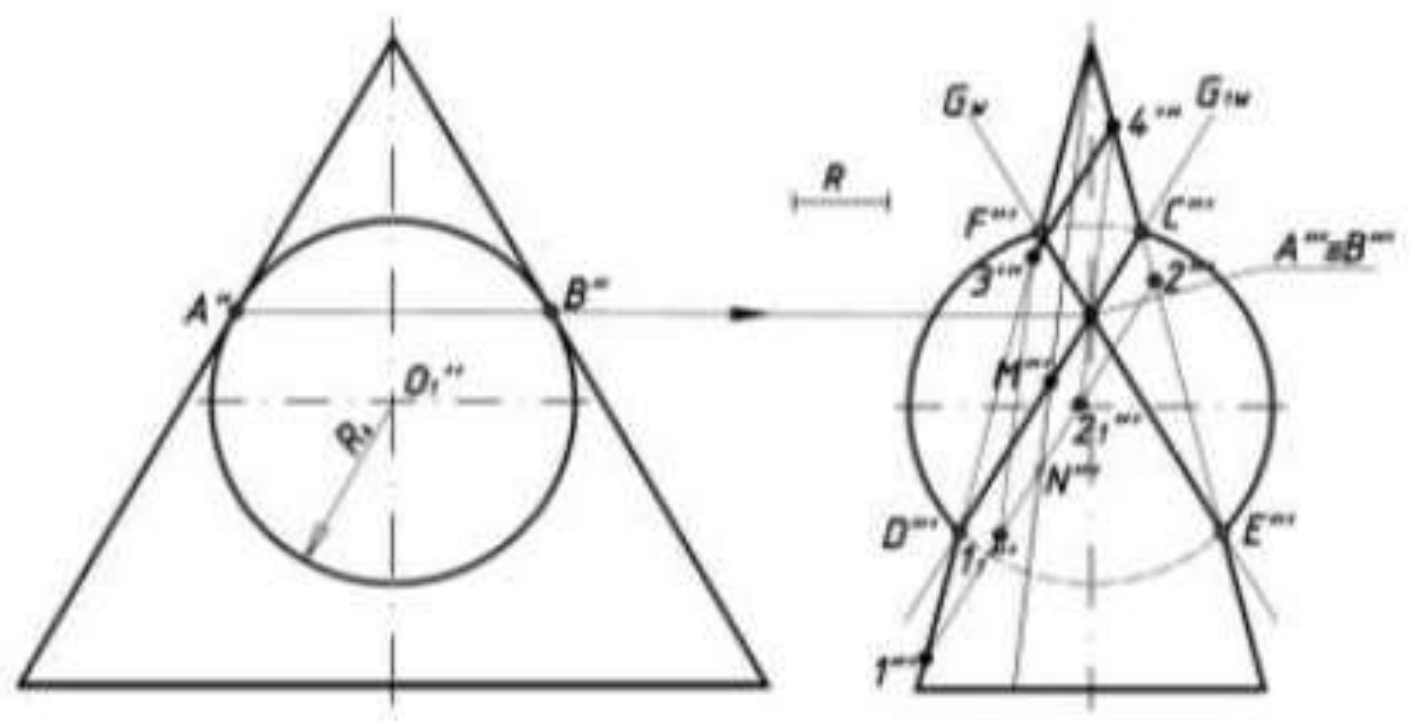

Figure 2.

\section{REFERENCES}

1. Analytical and graphic methods for solving inverse metric problems and their application in engineering practice 68 Art.

2. Shavkat Mirziyoyev. We will build our great future together with our brave and noble people.

3. Khorunov R. (1997). Course of descriptive geometry. Tashkent: "Teacher".

4. Khorunov R., Akbarov A. (1995). Problems from descriptive geometry and methods of their solution. Tashkent: "O'qituvchi”.

5. Murodov Sh ., Hakimov L., Odilov P., Shomurodov A., Jumaev M. (1998). Drawing geometry course. Tashkent: “O'qituvchi”.

6. Toshpulatov F. U., Buronov A. E., Abdukhairova M. Sh., Boboqulova Sh. Sh., Diyorova F. U. (2019). Comparison And Practical Results Of Two Promising
Methods. International Journal of Research, 6(13), 531-535.

7. Boltaboevich, B. B. (2020). Formation of the skills of portraying the future teacher of fine arts in pencil drawing. ACADEMICIA: An International Multidisciplinary Research Journal, 10(5), 1122-1127.

8. Boltabayevich, B. B., \& Shodievna, B. O. (2020). Individual Approach To The Formation Of Artistic And Creative Talents Of Students In Art Schools. The American Journal of Social Science and Education Innovations, 2(08), 637-642.

9. Baymetov, B. B. (2020). Art Of Modern Uzbekistan: The History Of Its Development During The Years of Independence. The American Journal of Social Science and Education Innovations, 2(10), 125-132. 
10. Karimov, N. R. (2020). A True Successor of Great Central Asian Scholars. Journal «Bulletin Social-Economic and Humanitarian Research,(7), 62-69.

11. Rasuljanovna, I. N., \& Rakhmonqulovich, K. N. (2020). Trade Relations Between Ancient Bacteria And China On The II-I BC. The American Journal of Social Science and Education Innovations, 2(07), 47-51.

12. Kariev, A., \& Aminov, H. (2020). New information about Imam al-Zarnūji and his work "Ta'lim al-Muta'allim". Solid State Technology, 63(6), 2372-2387. 